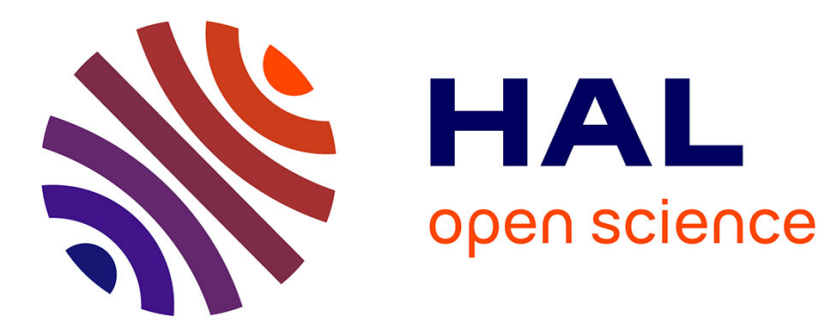

\title{
On stable rules for selecting committees Eric Kamwa
}

\section{- To cite this version:}

Eric Kamwa. On stable rules for selecting committees. Journal of Mathematical Economics, 2017, 70, pp.36 - 44. 10.1016/j.jmateco.2017.01.008 . hal-01631177

\section{HAL Id: hal-01631177 https://hal.univ-antilles.fr/hal-01631177}

Submitted on 27 Jun 2019

HAL is a multi-disciplinary open access archive for the deposit and dissemination of scientific research documents, whether they are published or not. The documents may come from teaching and research institutions in France or abroad, or from public or private research centers.
L'archive ouverte pluridisciplinaire HAL, est destinée au dépôt et à la diffusion de documents scientifiques de niveau recherche, publiés ou non, émanant des établissements d'enseignement et de recherche français ou étrangers, des laboratoires publics ou privés. 


\title{
On Stable Rules for Selecting Committees
}

\author{
Eric Kamwa
}

Université des Antilles, Faculté de Droit et d'Economie de la Martinique, Campus de Schoelcher, F-97275 Schoelcher and Laboratoire Caraïbéen de Sciences Sociales, LC2S UMR CNRS 8053

\begin{abstract}
A voting rule is said to be stable if it always elects a fixed-size subset of candidates such that there is no outside candidate who is majority preferred to any candidate in this set whenever such a set exists. Such a set is called a Weak Condorcet Committee (WCC). Four stable rules have been proposed in the literature. In this paper, we propose two new stable rules. Since nothing is known about the properties of the stable rules, we evaluate all the identified stable rules on the basis of some appealing properties of voting rules. We show that they all satisfy the Pareto criterion and they are not monotonic. More, we show that every stable rule fails the reinforcement requirement.
\end{abstract}

Keywords: Committee, Condorcet, Stable rule, Reinforcement axiom, Pareto criterion.

JEL classification: D70, D71

\section{Introduction}

Modern democracies use different voting rules (systems) for electing parliaments or groups (committees) of representatives. The most popular voting rules are, among others, the Plurality rule (used in India, in Great Britain, etc.), the Proportional system (used in Germany, Lebanon, etc). A committee or a group of representatives is a fixed-size set of alternatives (candidates) chosen from a larger set of contenders. Committees are chosen to fulfill a given purpose and their composition can be subject to some constraints or prerequisites: gender equity, minority representation, quotas and so on. Though a committee meets the prerequisites, it may happen that one wonders about the real legitimacy of this committee since different voting rules may lead to different outcomes. As the legitimacy of a committee does not depend only on its composition but also on the voting rule used, this gives rise to a couple of questions. What is a good committee? Does such a committee exist? What should be a good voting rule for selecting committees? Since the seminal works of Sterne (1871) and Dodgson (1885a,b, 1884, 1876), many political scientists and social choice theorists have tried to suggest how voting rules can be designed or used for selecting committees. See among others the works of Brams (2008), Brams et al (2005), Chamberlin and Courant (1983), Dummett (1984), Good and Tideman (1976), Hill (1988), Kilgour et al. (2006), Monroe (1995), Tullock (1967). A recent paper of Elkind et al. (2014) examined the properties of some voting rules in multi-winners context.

Email address: eric. kamwa@univ-antilles.fr (Eric Kamwa)

Preprint submitted to Journal of Mathematical Economics 
According to Gerhlein (1985), one of the prerequisites that can be imposed for committee selection is the fulfillment of the Condorcet criterion ${ }^{1}$ (Condorcet, 1785). For committee elections to be in line with the Condorcet criterion, Gerhlein (1985) suggested the selection, when it exists, of the fixed-size subset of candidates such that every member majority dominates every non-member: the Condorcet committee (à la Gerhlein). Such a set does not always exist in general (Gerhlein, 1985); when it exists it is unique (Good, 1971, Miller, 1980). Gerhlein (1985) computed the likelihood of such a set to exist using monte-carlo simulations. He found that with four contenders there is a $73.6 \%$ chance to end with a Condorcet committee of two members and $82.4 \%$ for a three-member committee; with seven contenders, there is a $35 \%$ chance to get a Condorcet committee of three members and $31.2 \%$ for a four-member committee. The likelihood of a Condorcet committee tends to decrease with the number of contenders and the size of the committee to be elected. Gerhlein (1985) concluded that almost all the well-known voting rules do not always select the Condorcet committee à la Gerhlein when it exists. Up to our knowledge, there are only two voting rules that have been suggested as always selecting the Condorcet committee (à la Gerhlein) when it exists. These rules suggested by Ratliff (2003) are:

- The Kemeny-Ratliff rule (KR), which is an adaptation of the Kemeny rule ${ }^{2}$, selects the subset of $g$ candidates with the smallest total margin of loss in pairwise comparisons versus the $m-g$ remaining candidates.

- The Dodgson-Ratliff rule (DR) is an adaptation of the Dodgson rule ${ }^{3}$. It selects the subset of $g$ candidates that requires the fewest number of adjacency switches to become a Condorcet committee à la Gerhlein.

Many recent papers have focused on the conditions that guarantee the existence of the Condorcet committee. See among others the works of Darmann (2013), Elkind et al. (2011, 2015a), Kamwa and Merlin (2013), Kaymak and Sanver (2003). What comes out from the results of these authors is that the Condorcet committee seems to be more restrictive as it is hard to get or to find $\mathrm{d}^{4}$. There is a version of the Condorcet committee that is less demanding and more likely to exist: the weak Condorcet Committee (WCC). A WCC is a fixed-size subset of candidates such that none of its members is defeated in pairwise comparisons by an outside candidate. It is obvious that a Condorcet committee (à la Gerhlein) is also a WCC; but a WCC is not necessarily a Condorcet committee (à la Gerhlein) $)^{5}$. Given that $g$ is the size of the committee to be elected, the WCC does not exist for some voting profiles while there may exist more than one WCC for some voting profiles.

\footnotetext{
${ }^{1}$ In one winner-election, this criterion requires that a candidate should be declared as the winner if he defeats each of the other candidates in pairwise comparisons; such a candidate is called the Condorcet winner.

${ }^{2}$ Given a preference profile with at least three candidates, the Kemeny rule (Kemeny, 1959, Kemeny and Snell, 1960) operates by computing distances from a given linear order to all the linear orders of the preference profile. The Kemeny ranking is the linear order that minimizes the total distance to the whole profile; the Kemeny winner is the candidate at the top of this ranking.

${ }^{3}$ The Dodgson rule (Dodgson, 1876) elects the candidate who requires the fewest number of switches (or adjacent switches of candidates) in voters' preferences in order to become the Condorcet winner. By an adjacent switch of $x$ and $y$ we mean swapping them in the given linear order. As shown by Bartholdi et al. (1989), Hemaspaandra et al. (1997), computing the Dodgson-scores is computationally intractable.

${ }^{4}$ Please refer to the work of Darmann (2013).

${ }^{5}$ There is also another definition of the Condorcet committee suggested by Fishburn (1981); please see the works of Kamwa and Merlin (2013), Kaymak and Sanver (2003) for the connections between the Fishburn's and the Gerhlein's definitions.
} 
According to Coelho (2004) ${ }^{6}$ a voting rule is said to be stable if it always selects a WCC when it exists. Coelho (2004) showed that almost all the well-known voting rules in the social choice literature and even those in use in the real life (such as the Plurality rule and the Borda rule) are not stable. Coelho (2004) concluded that the Kemeny-Ratliff rule and the Dodgson-Ratliff rule are also stable if they are used for the selection of $\mathrm{WCC}^{7}$. Coelho (2004) also suggested two other stable rules:

- The Minimal Number of External Defeats rule (NED) which selects the committee(s) of size $g$ for which the number of pairwise comparisons lost by its members is minimal.

- The Minimal Size of External Opposition rule (SEO) which is clearly an adaptation of the Maximin rule ${ }^{8}$ to committee elections. Given a committee of size $g$, its margin of loss is the highest margin of lost of a candidate in this committee against an outside candidate. The SEO rule elects the committee(s) with the smallest margin of loss.

Coelho (2004) argued that the Kemeny rule, the Dodgson rule and the Maximin rule are not stable when selecting committees by just appointing the best $g$ candidates of these rules. Kamwa (2014) came to a similar conclusion about the Young rule ${ }^{9}$. In this paper, we suggest two new stable rules:

- The Young-Condorcet rule (YC) which is adapted from the Young rule for committee elections. Given $g$ as the size of the committee to be elected, the YC rule will select the set of $g$ candidates that need the fewest number of deletions of voters to become a WCC.

- The Minimal Deletion of Candidates rule (MDC) which selects the set of $g$ candidates that need the fewest number of deletions of candidates to become a WCC.

We have to point out that if we want to select one-member committees with an odd number of voters, the KR rule is equivalent to the Kemeny rule, the DR rule to the Dodgson rule, the SEO rule to the Maximin rule and the YC rule to the Young rule. So, in this paper, our concern will be on committees of at least two members.

Even though four of the stable rules we focus on are adapted from well-known voting rules, nothing is known about their normative properties. Barberà and Coelho (2008) have shown that stability is incompatible with the property of enlargement consistency. Enlargement consistency requires that whenever a candidate is included in the chosen committee of size $g$, he must also be in the chosen committee of size $g+1$. As a first step toward a characterization of the whole family of stable rules, we evaluate our stable rules on the basis of some appealing properties of voting rules: the Condorcet winner criterion, the Condorcet loser criterion, the Pareto criterion,

\footnotetext{
${ }^{6}$ See also the paper of Barberà and Coelho (2008).

${ }^{7}$ The original KR rule is roughly applied; the DR rule will select the subset of $g$ candidates that requires the fewest number of adjacency switches to make this subset become a WCC.

${ }^{8}$ Given a voting situation, the Maximin rule (also called the Simpson-Kramer rule (Kramer, 1977, Simpson, 1969) or the Minimax rule (Young, 1977)), first determines the support received by each candidate in every pairwise comparison; the candidate with the greatest minimum support received is the winner. This rule can be traced back to Condorcet (1785) (see also the book of Black, 1958).

${ }^{9}$ Suggested by Young (1977), this rule proceeds by deletions of voters. The Young rule elects the candidate(s) who needs the fewest number of deletions of voters to become the Condorcet winner. Computing the Young-scores is computationally intractable: see among others the works of Rothe et al. (2003), Betzler et al. (2010) and Caragiannis et al. (1998).
} 
the monotonicity criterion, the homogeneity criterion and the reinforcement criterion. All these criteria are defined later.

The rest of the paper is structured as follows: Section 2 sets the framework with basic definitions. The formal definitions of the stable rules are provided in Section 3. In Section 4, we proceed to the evaluation of our stable rules. Section 5 concludes.

\section{Binary relations and preferences}

Let $N$ be the set of $n$ voters $(n \geq 2)$ and $A$ the set of $m$ candidates $(m \geq 3)$. A binary relation $R$ over $A$ is a subset of the cartesian product $A \times A$. For $a, b \in A$, if $\{a, b\} \in R$, we note $a R b$ to say " $a$ is at least as good as $b$ ". $\neg a R b$ is the negation of $a R b$. If we have $a R b$ and $\neg b R a$, we will say " $a$ is better or strictly preferred to $b$ ". In this case, we write $a P b$ with $P$ denoting the asymmetric component of $R$. The symmetric component of $R, I$, is defined by aIb denoting an indifference between $a$ and $b$, i.e, $a R b$ and $b R a$. The preference profile $\pi=\left(P_{1}, P_{2}, \ldots, P_{i}, \ldots, P_{n}\right)$ gives all the linear orders ${ }^{10}$ of all the $n$ voters on $A$, where $P_{i}$ is the strict ranking of a given voter $i$. The set of all the preference profiles of size $n$ on $A$ is denoted by $P(A)^{n}$. In the sequel, we will simply write, $a b c$ to denote that candidate $a$ is ranked before candidate $b$ who is ranked before $c$. A voting situation $\tilde{n}=\left(n_{1}, n_{2}, \ldots, n_{t}, \ldots, n_{m !}\right)$ indicates the number of voters for each linear order such that $\sum_{t=1}^{m !} n_{t}=n$

For a given profile $\pi$, when the number of voters who rank $a$ before $b$ (denoted by $n_{a b}$ ) is greater than that of those who rank $b$ before $a$ (denoted by $n_{b a}$ ), $a$ is said to be majority preferred to $b$. This can graphically be represented by an arrow going from $a$ to $b$. We denote this by $a M(\pi) b$ or simply $a M b$ when there is no ambiguity. For $a M b$, we say that candidate $b$ loses the majority contest by a margin equal to $n_{a b}-n_{b a}$. If $n_{a b}=n_{b a}$, we say that $a$ and $b$ tie and we denote it by $a T(\pi) b$ or simply $a T b$. Graphically, there will be no arrow between $a$ and $b$ when $a T b$. Given $\pi$, candidate $a$ is the Condorcet winner if we have $a M(\pi) b$ for all $b \in A \backslash\{a\}$. Candidate $a$ is the Condorcet loser if we have $b M(\pi) a$ for all $b \in A \backslash\{a\}$.

Definition 1. (Majority cycle). Let $B$ be a nonempty subset of $A$ such that $B=\left\{a_{1}, \ldots, a_{j}, \ldots . a_{l}\right\}$ with $3 \leq l \leq m$. Given the profile $\pi$, the majority relation $M$ is cyclic on $B$ if $a_{j} M a_{j+1} \forall j=$ $1,2, \ldots, l-1$ and $a_{l} M a_{1} ; l$ is the length of the cycle.

Assume that we want to elect a committee of size $g(1 \leq g \leq m-1)$. We denote by $\mathcal{A}^{g}$ the set of all possible committees of size $g$. A Weak Condorcet Committee (WCC) is a fixed size subset of candidates such that no candidate in this subset is defeated in pairwise comparisons by any outside candidate. Throughout the paper, we adopt the notation $\{a, b\}$ to refer to the committee made of candidates $a$ and $b$.

Definition 2. (Weak Condorcet Committee) With $|A|=m, C \in \mathcal{A}^{g}$ is a WCC if and only if $\forall x \in C$ and $\forall y \in A \backslash C$ we do not have $y M x$.

Given a voting situation, a WCC may not exist for a given $g$ (see Example 1). For some voting situations, we can have one or more WCC given $g$ (see Example 2 ). We denote by $C_{g}(\pi)$ the set of all the WCC of size $g$ given the profile $\pi$.

\footnotetext{
${ }^{10} \mathrm{~A}$ linear order is a binary relation that is transitive, reflexive, complete and antisymmetric. A binary relation $R$ on $A$ is transitive if for $a, b, c \in A$, if $a R b$ and $b R c$ then $a R c . R$ is reflexive if for all $a \in A$, one can write $a R a$. $R$ is complete if and only if for all $a, b \in A$, we have $a R b$ or $b R a$. $R$ is antisymmetric if for all $a, b \in A, a R b \Rightarrow \neg b R a$.
} 
Example 1. Consider the following preference profile $\pi_{1}$ with 3 voters on $A=\{a, b, c, d\}$.

\begin{tabular}{ccc}
\multicolumn{3}{c}{ profile $\pi_{1}$} \\
\hline $1:$ acbd & $1:$ bdca & $1:$ dcba \\
\hline \hline
\end{tabular}

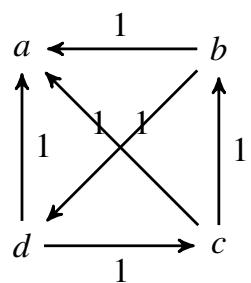

It comes that $b M\left(\pi_{1}\right) a, c M\left(\pi_{1}\right) b, b M\left(\pi_{1}\right) d, d M\left(\pi_{1}\right) a, d M\left(\pi_{1}\right) c$ and $c M\left(\pi_{1}\right) a$. So, $C_{2}\left(\pi_{1}\right)=\emptyset$ while $C_{3}\left(\pi_{1}\right)=\{b, c, d\}$.

Example 2. Consider the following preference profile $\pi_{2}$ with 6 voters on $A=\{a, b, c, d\}$.

\begin{tabular}{ccc}
\multicolumn{4}{c}{ profile $\pi_{2}$} \\
\hline $1: b c d a$ & $1:$ cabd $\quad 3: a d b c$ & $1:$ bacd \\
\hline \hline
\end{tabular}

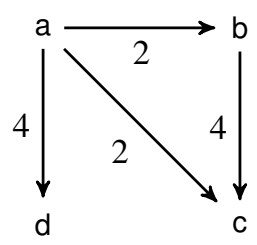

With this profile, we have $a M\left(\pi_{2}\right) b, a M\left(\pi_{2}\right) c, a M\left(\pi_{2}\right) d, b M\left(\pi_{2}\right) c, b T\left(\pi_{2}\right) d$ and $c T\left(\pi_{2}\right) d$. So, for $g=2$ we get $C_{2}\left(\pi_{2}\right)=\{\{a, b\},\{a, d\}\} ;$ for $g=3$ we get $C_{3}\left(\pi_{2}\right)=\{\{a, b, c\},\{a, b, d\}\}$.

Let us now define each of the stable rules we focus on.

\section{The stable rules}

In this section, we first provide a formal definition of each of the stable rules we focus on; then, we apply these rules to a voting profile (in Example 3). Given a profile $\pi$ with $|A|=m \geq 3$, $\mathcal{A}^{g}$ the set of all possible committees of size $g$ and a committee $C \in \mathcal{A}^{g}$,

- The Kemeny-Ratliff rule (KR) selects the subset of $g$ candidates with the smallest total margin of loss in pairwise comparisons versus the $m-g$ remaining candidates. The KRscore of a committee $C$ denoted $K R(\pi, C)$ is given by : $K R(\pi, C)=\sum_{x \in C, y \in A \backslash C} \max \left\{0, n_{y x}-\right.$ $\left.n_{x y}\right\}$ and the KR outcome set, $\mathcal{K R}_{g}(\pi)$ is defined as follows: $\mathcal{K} \mathcal{R}_{g}(\pi)=\left\{C \in \mathcal{A}^{g}\right.$ : $\left.K R(\pi, C) \leq K R\left(\pi, C^{\prime}\right) \forall C^{\prime} \in \mathcal{A}^{g} \backslash\{C\}\right\}$.

- The Dodgson-Ratliff rule (DR) chooses the subset(s) of $g$ candidates that requires the fewest number of adjacency switches to make this subset become a weak Condorcet committee à la Gerhlein (WCC). The number of switches needed to become a WCC defines the DR-score of committee $C$ denoted $D R(\pi, C)$. The DR outcome set, denoted $\mathcal{D} \mathcal{R}_{g}(\pi)$, is $\mathcal{D R}_{g}(\pi)=\left\{C \in \mathcal{A}^{g}: D R(\pi, C) \leq D R\left(\pi, C^{\prime}\right) \forall C^{\prime} \in \mathcal{A}^{g} \backslash\{C\}\right\}$.

- The Minimal Size of External Opposition rule (SEO) elects the committee(s) with the smallest margin of loss of its members. The SEO-score of committee $C$, denoted $\operatorname{SEO}(\pi, C)$, is given by : $\operatorname{SEO}(\pi, C)=\max _{x \in C, y \in A \backslash C} n_{y x}$. The $\mathrm{SEO}$ outcome set, $\mathcal{S E O}_{g}(\pi)$ is : $\mathcal{S E O}_{g}(\pi)=$ $\left\{C \in \mathcal{A}^{g}: \operatorname{SEO}(\pi, C) \leq \operatorname{SEO}\left(\pi, C^{\prime}\right) \forall C^{\prime} \in \mathcal{A}^{g} \backslash\{C\}\right\}$ 
- The Minimal Number of External Defeats Rule (NED) selects the committee(s) of size $g$ for which the number of pairwise comparisons lost by its members is minimal. The NEDscore of committee $C$, denoted $\operatorname{NED}(\pi, C)$, is given by : $\operatorname{NED}(\pi, C)=\sum \mid\left\{y \in A \backslash C: n_{x y}<\right.$ $\left.n_{y x}\right\} \mid$. The NED outcome set, denoted $\mathcal{N E D}_{g}(\pi)$, is $\mathcal{N E}_{\mathcal{E}} \mathcal{D}_{g}(\pi)=\left\{C \in \mathcal{A}^{g}: \operatorname{NED}(\pi, C) \leq\right.$ $\left.\operatorname{NED}\left(\pi, C^{\prime}\right) \quad \forall C^{\prime} \in \mathcal{A}^{g} \backslash\{C\}\right\}$.

- The Young-Condorcet rule $(Y C)$ selects the set of $g$ candidates that need the fewest number of deletions of voters to become a WCC. Let $\pi^{S}$ be the profile obtained after the deletion of a subset $S$ of voters $(S \subseteq N)$. The YC-score of a committee $C$ is given by : $Y C(\pi, C)=$ $\min _{S \in \Delta(\pi, C)}|S|$; with $\Delta(\pi, C)=\left\{S \subseteq N: C \in C_{g}\left(\pi^{S}\right)\right\}$. The YC outcome set, denoted $\mathcal{Y C}_{g}(\pi)$, is defined as follows : $\mathcal{Y C}_{g}(\pi)=\left\{C \in \mathcal{A}^{g}: Y C(\pi, C) \leq Y C\left(\pi, C^{\prime}\right) \forall C^{\prime} \in \mathcal{A}^{g} \backslash\{C\}\right\}$.

- The Minimal number of Deletion of Candidates $(M D C)$ elects the set of $g$ candidates that need the fewest number of deletions of candidates to become a WCC. Given $B$ a subset of $A$, let $\pi_{B}$ be a voting profile obtained after the deletion of a subset $B$ of candidates. The MDC-score of a committee $C \in \mathcal{A}^{g}$ is given by : $\operatorname{MDC}(\pi, C)=\min _{B \in \Omega(\pi, C)}|B|$; with $\Omega(\pi, C)=\left\{B \subseteq A: C \in C_{g}\left(\pi_{B}\right)\right\}$. The MDC outcome set, $\mathcal{M D C} C_{g}(\pi)$, is defined as follows $: \mathcal{M D C}_{g}(\pi)=\left\{C \in \mathcal{A}^{g}: \operatorname{MDC}(\pi, C) \leq \operatorname{MDC}\left(\pi, C^{\prime}\right) \forall C^{\prime} \in \mathcal{A}^{g} \backslash\{C\}\right\}$.

In order to illustrate our stable rules, let us consider a voting profile drawn from Ratliff (2003, p.436).

Example 3. Consider a profile with $A=\{a, b, c, d\}$ and $N=33$.

\begin{tabular}{cc}
\multicolumn{2}{c}{ preferences } \\
\hline \hline $6: a b c d$ & $10: c d b a$ \\
$5: b c d a$ & $1: b a c d$ \\
$10: a d b c$ & $1: d c a b$ \\
\hline \hline
\end{tabular}

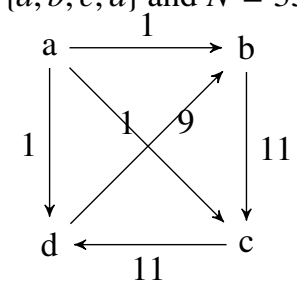

\begin{tabular}{|c|c|c|c|c|}
\hline \multicolumn{5}{|c|}{ Pairwise comparisons } \\
\hline vs & $a$ & $b$ & $c$ & $d$ \\
\hline$a$ & - & 17 & 17 & 17 \\
\hline$b$ & 16 & - & 22 & 12 \\
\hline$c$ & 16 & 11 & - & 22 \\
\hline$d$ & 16 & 21 & 11 & - \\
\hline
\end{tabular}

After all computations, we report the scores for $g=2$ and $g=3$ as it follows:

\begin{tabular}{|c|c|c|c|c|c|c|}
\cline { 2 - 7 } \multicolumn{1}{c|}{} & \multicolumn{7}{c|}{ Scores for $g=2$} \\
\hline committees & KR & DR & SEO & NED & YC & MDC \\
\hline$\{a, b\}$ & 9 & 5 & 21 & 1 & 9 & 1 \\
\hline$\{a, c\}$ & 11 & 6 & 22 & 1 & 11 & 1 \\
\hline$\{a, d\}$ & 11 & 6 & 22 & 1 & 11 & 1 \\
\hline$\{b, c\}$ & 11 & 7 & 21 & 3 & 9 & 2 \\
\hline$\{b, d\}$ & 13 & 7 & 22 & 3 & 11 & 2 \\
\hline$\{c, d\}$ & 13 & 7 & 22 & 3 & 11 & 2 \\
\hline \multicolumn{7}{|c|}{ Scores for $g=3$} \\
\hline committees & KR & DR & SEO & NED & YC & MDC \\
\hline$\{a, b, c\}$ & 9 & 5 & 21 & 1 & 9 & 1 \\
\hline$\{a, b, d\}$ & 11 & 6 & 22 & 1 & 11 & 1 \\
\hline$\{a, c, d\}$ & 11 & 6 & 22 & 1 & 11 & 1 \\
\hline$\{b, c, d\}$ & 3 & 3 & 17 & 3 & 1 & 1 \\
\hline
\end{tabular}

- For $g=2$, we get $\mathcal{K} \mathcal{R}_{2}(\pi)=\mathcal{D R}_{2}(\pi)=\{a, b\}, \mathcal{S E O}_{2}(\pi)=\mathcal{Y C}_{2}(\pi)=\{\{a, b\},\{b, c\}\}$ and $\mathcal{N E D}_{2}(\pi)=\mathcal{M D C}_{2}(\pi)=\{\{a, b\},\{a, c\},\{a, d\}\}$. 
- For $g=3$, we get $\mathcal{K} \mathcal{R}_{3}(\pi)=\mathcal{D R}_{3}(\pi)=\mathcal{S E O}_{3}(\pi)=\mathcal{Y C}_{3}(\pi)=\{b, c, d\}, \mathcal{N E D}_{3}(\pi)=$ $\{\{a, b, c\},\{a, b, d\},\{a, c, d\}\}$ and $\mathcal{M D C}_{3}(\pi)=\{\{a, b, c\},\{a, b, d\},\{a, c, d\},\{b, c, d\}\}$.

We admit that aside from the six stable rules analyzed in this paper, there may exist other stable rules. To all intents and purposes, note that we are not concerned here by the complexity of these rules since this question is out of our field of competence. Notice that the NED rule has a Copeland-like flavor. According to Coelho (2004) and Kamwa (2014), the rule that chooses the best $g$ Copeland winners and all the Copeland-like flavor's rules that iteratively selects a Copeland winner until $g$ candidates have been selected are not stable. We know from Ratliff (2003) that a committee selected by the KR rule (resp. the DR rule) is not always made of the top $g$ candidates of the Kemeny (resp. the Dodgson) ranking. This is also the case concerning the YC rule and the Young ranking, the SEO rule and the Maximin ranking. Therefore there is no formal connection between a stable rule and the classical rule it is derived from. Kamwa $(2016,2014)$ showed that in three-candidate elections and two-member committees, the outcome set of the KR rule, the DR rule, the YC rule and the SEO rule is always the same while the NED rule and the MDC rule select the whole set of two-member committees. Let us now check some properties of our stable rules.

\section{Some properties of the stable rules}

We have mentioned that the KR rule, the DR rule, the SEO rule and the YC rule are adapted from well-known voting rules. While many things are known regarding the properties ${ }^{11}$ of the Kemeny rule, the Dodgson rule, the Maximin rule and the Young rule almost nothing is known concerning the corresponding stable rules. From Barberà and Coelho (2008), we know that there is no stable rule satisfying enlargement consistency. Recall that a voting rule satisfies enlargement consistency whenever a candidate who is included in an elected committee of size $g$ must also be in the elected committee of $\operatorname{size}^{12} g+1$. What we want to do in this section is to evaluate our stable rules on the basis of six important criteria ${ }^{13}$ : the Condorcet winner criterion, the Condorcet loser criterion, the Pareto criterion, the monotonicity criterion, the reinforcement criterion and the homogeneity criterion. We will redefine some of these properties in order to suit to the framework of committee elections.

\subsection{The Condorcet criterion}

As noticed, the notion of "Condorcet committee" is built on the Condorcet winner criterion. It seems natural to check how our stable rules behave when there is a Condorcet winner or a Condorcet loser.

A Condorcet winner is a candidate who defeats each of the other ones in pairwise contests. In a one-winner election, a voting rule is said to be Condorcet consistent or to satisfy the Condorcet winner criterion if it always elects the Condorcet winner when he exists. It is known that the Kemeny rule, the Dodgson rule, the Maximin rule and the Young rule all satisfy the Condorcet

\footnotetext{
${ }^{11}$ See the works of Fishburn (1977), Nurmi (1987), Richelson (1979, 1981), Tideman (1987), Young and Levenglick (1978).

${ }^{12}$ When a voting rule fails to meet the enlargement consistency, this defines what Staring (1986) called the increasing committee size paradox. Kamwa (2013b), Mitchell and Trumbull (1992) focused on the likelihood of this paradox. This condition of enlargement consistency is called "Committee monotonicity" in Elkind et al. (2014).

${ }^{13}$ Elkind et al. (2014) performed a remarkable evaluation of many other voting rules for committee elections.
} 
winner criterion. If this criterion is applied to committee elections it will require that when there is a Condorcet winner, he must belong to the elected committee. We know that the Condorcet winner when he exists always belongs to the WCC if there is one. To know if our stable rules satisfy the Condorcet winner criterion, we have to check how they behave when there is a Condorcet winner and no WCC. Lemma 1 tells us that when there is no WCC and that there is a Condorcet winner, the elected committee under the NED rule and the MDC rule always contains the Condorcet winner.

Lemma 1. Consider a profile $\pi$ with $m \geq 3$ and $2 \leq g \leq m-1$ such that $C_{g}=\emptyset$ and there is Condorcet winner. The NED-score and the MDC-score are always minimized by a committee that contains the Condorcet winner.

Proof. Consider a profile $\pi$ with $m \geq 3$ and given $2 \leq g \leq m-1$ such that $C_{g}=\emptyset$. Assume in this profile that there is a Condorcet winner $x \in A$ and that there is a committee $C \in \mathcal{N E D}_{g}(\pi)$ such that $x \notin C$. Given $y \in C$ we define a committee $C^{\prime}=C \backslash\{y\} \cup\{x\}$. Let us define $Z=C \cap C^{\prime}$ and $Q=A \backslash\left\{C \cup C^{\prime}\right\}$.

We denote by $\operatorname{def}(y / Q)$ the total number of defeats of candidate $y$ versus candidates in $Q$. Similarly, we define $\operatorname{def}(Z / Q)$ the total number of defeats of candidates in $Z$ versus those in $Q$. In the same manner we $\operatorname{define} \operatorname{def}(y / x)$ and $\operatorname{def}(Z / y)$. Then, we get

$$
\begin{aligned}
\operatorname{NED}(\pi, C) & =\operatorname{def}(y / x)+\operatorname{def}(Z / x)+\operatorname{def}(y / Q)+\operatorname{def}(Z / Q) \\
\operatorname{NED}\left(\pi, C^{\prime}\right) & =\operatorname{def}(Z / y)+\operatorname{def}(Z / Q)
\end{aligned}
$$

By definition, $\operatorname{def}(y / x)+\operatorname{def}(Z / x)=g$. It follows that $\operatorname{NED}(\pi, C)-\operatorname{NED}\left(\pi, C^{\prime}\right)=g+\operatorname{def}(y / Q)-$ $\operatorname{def}(Z / y)$. Since $0 \leq \operatorname{def}(Z / y) \leq g-1$, it comes that $\operatorname{NED}(\pi, C)-\operatorname{NED}\left(\pi, C^{\prime}\right)>0 \Leftrightarrow \operatorname{NED}(\pi, C)>$ $\operatorname{NED}\left(\pi, C^{\prime}\right)$. So, the NED-score is always minimized by a committee that contains the Condorcet winner.

Let us assume that $C \in \mathcal{M D C} C_{g}(\pi)$. As $x$ is the Condorcet winner, he certainly belongs to the subset of candidates to be deleted in order to get $C \in \mathcal{M D C} C_{g}(\pi)$. For simplicity, we denote by $B(Q / Z)$ the subset of candidates in $Q$ that defeat some candidates in $Z$ and by $B(Q / y)$ the subset of candidates in $Q$ that defeat $y$. It follows that for committee $C$ we get $\operatorname{MDC}(\pi, C)=\mid B(Q / Z) \cup$ $B(Q / y) \cup\{x\} \mid$; for committee $C^{\prime}$ we get $\operatorname{MDC}\left(\pi, C^{\prime}\right)=|B(Q / Z)|$ or $\operatorname{MDC}\left(\pi, C^{\prime}\right)=|B(Q / Z) \cup\{y\}|$. As there is no WCC, it comes that $\operatorname{MDC}(\pi, C) \geq \operatorname{MDC}\left(\pi, C^{\prime}\right)$. Therefore the MDC rule meets the Condorcet winner criterion.

In Example 3, candidate $a$ is the Condorcet winner but he is not in $\{b, c, d\}$ which is the elected committee (for $g=3$ ) for the DR rule, the KR rule, the SEO rule and the YC rule. Therefore these rules do not meet the Condorcet winner criterion. Theorem 1 states that if there is a Condorcet winner, among our six stable rules, only the NED rule and the MDC rule always satisfy the Condorcet winner criterion no matter the size of the committee to be elected.

Theorem 1. Given $m \geq 3$ and $2 \leq g \leq m-1$, the NED rule and the MDC rule always select committees that contain the Condorcet winner.

The proof of Theorem 1 follows directly from Lemma 1.

A Condorcet loser is a candidate who is defeated in all pairwise contests. In one-winner elections, a voting rule is said to meet the Condorcet loser criterion if it never elects the Condorcet loser when he exists. Obviously, when a Condorcet loser exists he does not belong to the WCC if there is one. In committee elections, we say that a voting rule satisfies the Condorcet loser 
criterion if when there is Condorcet loser, this candidate cannot belong to an elected committee. Theorem 2 tells us that among the stable rules analyzed here only the NED and the MDC rules satisfy the Condorcet loser criterion.

Theorem 2. With $m \geq 3$ candidates and $2 \leq g \leq m-1$, among the stable rules in concern here only the NED and the MDC rules always prevent the election of the Condorcet loser when he exists.

Proof. To check how our stable rules behave in the presence of a Condorcet loser, we suppose that there is no WCC for a given $g$. Let us use a profile to show that the KR rule, the DR rule, the SEO rule and the YC rule fail the Condorcet loser criterion. Consider the following profile with 21 voters and 5 candidates.

\begin{tabular}{l} 
rankings \\
\hline \hline $7: a e d b c$ \\
$3:$ aecdb \\
$7:$ bcdea \\
$4:$ cdbea \\
\hline \hline
\end{tabular}

Pairwise comparisons
\begin{tabular}{|c|c|c|c|c|c|}
\hline \hline vs & $a$ & $b$ & $c$ & $d$ & $e$ \\
\hline$a$ & - & 10 & 10 & 10 & 10 \\
\hline$b$ & 11 & - & 14 & 7 & 11 \\
\hline$c$ & 11 & 7 & - & 14 & 11 \\
\hline$d$ & 11 & 14 & 7 & - & 11 \\
\hline$e$ & 11 & 10 & 10 & 10 & - \\
\hline \hline
\end{tabular}

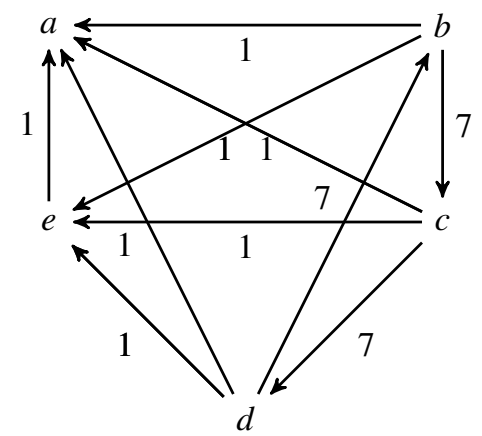

In this profile, candidate $a$ is the Condorcet loser. After all computations, we get $\mathcal{K} \mathcal{R}_{2}(\pi)=$ $\mathcal{D R}_{2}(\pi)=\mathcal{Y C}_{2}(\pi)=\mathcal{S E O}_{2}(\pi)=\{a, e\}$. We see that candidate $a$ belongs to the elected committee. We then conclude that the KR rule, the DR rule, the SEO rule and the YC rule do not meet the Condorcet loser criterion.

Now, let us prove that a committee that contains the Condorcet loser is never included in the NED set. Consider a profile $\pi$ with $m \geq 3$ and $2 \leq g \leq m-1$ such that $C_{g}=\emptyset$. Assume in this profile that there is a Condorcet loser $x \in A$ and that there is a committee $C \in \mathcal{N E D}_{g}(\pi)$ such that $x \in C$. We define a committee $C^{\prime}=C \backslash\{x\} \cup\{y\}$ with $y \in A \backslash C$. Let us take $Z=C \cap C^{\prime}$ and $Q=A \backslash\left\{C \cup C^{\prime}\right\}$. We denote by $\operatorname{def}(y / Q)$ the total number of defeats of candidate $y$ versus candidates in $Q$. Similarly, we $\operatorname{define} \operatorname{def}(Z / Q)$ the total number of defeats of candidates in $Z$ versus those in $Q$. If in the same manner we $\operatorname{define} \operatorname{def}(x / y)$ and $\operatorname{def}(Z / y)$. We get

$$
\begin{aligned}
\operatorname{NED}(\pi, C) & =\operatorname{def}(x / y)+\operatorname{def}(x / Q)+\operatorname{def}(Z / y)+\operatorname{def}(Z / Q) \\
\operatorname{NED}\left(\pi, C^{\prime}\right) & =\operatorname{def}(y / Q)+\operatorname{def}(Z / Q)
\end{aligned}
$$

By definition $\operatorname{def}(x / y)=1$ and we get $\operatorname{NED}(\pi, C)-\operatorname{NED}\left(\pi, C^{\prime}\right)=1+\operatorname{def}(Z / y)+|Q|-\operatorname{def}(y / Q)$. Since $0 \leq \operatorname{def}(y / Q) \leq|Q|$, it comes that $\operatorname{NED}(\pi, C)-\operatorname{NED}\left(\pi, C^{\prime}\right)>0 \Leftrightarrow \operatorname{NED}(\pi, C)>$ $\operatorname{NED}\left(\pi, C^{\prime}\right)$. This contradicts that $C \in \mathcal{N} \mathcal{E} \mathcal{D}_{g}(\pi)$.

Concerning the MDC rule, it is obvious that $\operatorname{MDC}(\pi, C)=m-g$ while $\operatorname{MDC}\left(\pi, C^{\prime}\right)<m-g$; this contradicts that $C \in \mathcal{M D C}(\pi)$. Therefore the MDC rule meets the Condorcet loser criterion.

\subsection{The Pareto criterion}

A voting procedure is said to satisfy the Pareto criterion if it never elects a candidate $x$ while there is a candidate $y$ that all the voters rank before $x$. According to Felsenthal (2012), there 
seems to be a wide consensus that a voting procedure that may lead to a Pareto-dominated candidate should be disqualified regardless of how often this can happen. In our context of committee elections, the Pareto criterion will require that if there are two candidates $x$ and $y$ such that $x$ is always ranked ahead of $y$, then if there is a winning committee that includes $y$, then there is also one that includes $x$.

assume that there is candidate $y$ that Pareto-dominates $x$; a selection rule will meet the Pareto criterion if when $x$ belongs to a selected committee this is also the case for $y$.

Theorem 3. Given $m \geq 3$ and $1 \leq g \leq m-1, K R$ rule, the DR rule, the MDC rule, the SEO rule, the NED rule and the YC rule meet the Pareto criterion.

Proof. See Appendix.

\subsection{Monotonicity}

In one-winner elections, a voting rule is said to be monotonic if when some voters decide to lift up a winning candidate $x$ in their rankings without changing anything else, this would not harm candidate $x$.

For committee elections, many definitions of the monotonicity are possible. One can conceive monotonicity in the sense that lifting up a candidate (member of an elected committee) will not push this candidate out of the set of the elected candidates no matter what is the new set of elected committees. We call this, the Candidate Monotonicity criterion. Another possible definition will say that a stable rule satisfies the Membership Monotonicity criterion if when some voters decide to lift up a candidate (member of an elected committee) in their rankings, without changing anything else, this would not be harmful to the committee to which this candidate belongs to i.e the considered elected committee could not be pushed out of the set of the elected committees after the lifting up.

In this paper, we only deal with the Candidate Monotonicity criterion and the Membership Monotonicity criterion as defined above.

Theorem 4. The KR rule, the DR rule, the MDC rule, the SEO rule, the NED rule and the YC rule satisfy the Candidate Monotonicity criterion ${ }^{14}$. With at least three voters and at least three candidates, all these stable rules fail the Membership Monotonicity criterion.

See Appendix for the proof of Theorem 4. At this stage of the analysis, we are not able to say whether the violation of the Membership Monotonicity criterion is just a rare oddity or betray a more generalized behavior. One way to do this is to follow the common approach in the social choice literature through probability computations.

\subsection{Homogeneity}

A voting rule is homogeneous if given a preference profile and the corresponding outcome, replicating this profile $\lambda$ times $(\lambda>1, \lambda \in \mathbb{N})$ does not change the outcome. According to Nurmi (2004, p.12) "inhomogeneous systems pose a major challenge to representative arrangements since the outcomes ensuing from the representative body depend not only on the correspondence between the voters' and their representatives' views but also on the size of the representative body". In our framework, we will say that a stable rule is homogeneous if whenever we replicate $\lambda$ times the original profile, the outcome set remains unchanged.

${ }^{14}$ This criterion is due to Elkind et al. (2014). 
Definition 3. (Homogeneity) Given that $g$ is the size of the committee to be elected and $V$ a stable rule. For $\mathcal{V}_{g}(\pi)$ the set of the elected committees with the profile $\pi$, the stable rule $V$ is homogeneous if and only if

$$
\mathcal{V}_{g}(\lambda \pi)=\mathcal{V}_{g}(\pi) \quad \forall \lambda>1, \lambda \in \mathbb{N}
$$

Theorem 5. Given $m \geq 4$ and $1 \leq g \leq m-1$, the KR rule, the SEO rule, the NED rule and the $M D C$ rule are homogeneous while the $Y C$ rule and the DR rule are not.

Proof. Given a profile $\pi$ and a committee $C$, for $\lambda>1$ we have by definition

$$
\operatorname{KR}(\lambda \pi, C)=\sum_{x \in C, y \in A \backslash C} \max \left\{0, \lambda n_{y x}-\lambda n_{x y}\right\}=\lambda \sum_{x \in C, y \in A \backslash C} \max \left\{0, n_{y x}-n_{x y}\right\}=\lambda \operatorname{KR}(\pi, C)
$$

So, the $\mathrm{KR}$ rule is homogeneous. Similarly we get $\operatorname{SEO}(\lambda \pi, C)=\lambda \operatorname{SEO}(\pi, C)$ : the $\operatorname{SEO}$ rule is homogeneous. By duplicating the electorate, this does not alter the number of defeats of a candidates; we get $\operatorname{NED}(\lambda \pi, C)=\operatorname{NED}(\pi, C)$ : so, the NED rule is homogeneous. When we replicate the electorate, the number of deletions of candidates in order to transform a given candidate into a WCC does not change; then we get $\operatorname{MDC}(\lambda \pi, C)=\operatorname{MDC}(\pi, C)$. So, the $\operatorname{MDC}$ rule is homogeneous.

Let us now show that the DR rule is not homogeneous. Consider the following profile with five candidates $A=\{a, b, c, d, e\}$ and 15 voters.

\begin{tabular}{ll}
\multicolumn{2}{c}{ rankings } \\
\hline \hline $3: e a b c d$ & $1: e a b d c$ \\
$4: e c a d b$ & $1: e c d a b$ \\
$4: e b d c a$ & $1: e d b c a$ \\
$1: e d a b c$ & \\
\hline \hline
\end{tabular}

\begin{tabular}{c|c|c|c|c|c|}
\multicolumn{7}{c|}{ Pairwise comparisons } \\
\hline \hline$v s$ & $a$ & $b$ & $c$ & $d$ & $e$ \\
\hline$a$ & - & 10 & 5 & 8 & 0 \\
\hline$b$ & 5 & - & 10 & 8 & 0 \\
\hline$c$ & 10 & 5 & - & 8 & 0 \\
\hline$d$ & 7 & 7 & 7 & - & 0 \\
\hline$e$ & 15 & 15 & 15 & 15 & - \\
\hline
\end{tabular}

The reader can check that with this profile we get $\mathcal{D} \mathcal{R}_{2}(\pi)=\{\{a, e\},\{b, e\},\{c, e\},\{d, e\}\}$. If we replicate the electorate 2 times, we get $\mathcal{D} \mathcal{R}_{2}(2 \pi)=\{d, e\}$; committees $\{a, e\},\{b, e\}$ and $\{c, e\}$ are no more appointed. Therefore the DR rule is not homogeneous.

In order to show that the YC rule is not homogeneous ${ }^{15}$, let us consider the following profile adapted from Young (1977) with seven candidates $A=\{a, b, c, d, e, f, g\}$ and 9 voters.

\begin{tabular}{lr}
\multicolumn{2}{c}{ rankings } \\
\hline \hline $1:$ geabcdf & $1:$ gabcefd \\
$1:$ gabecfd & $1:$ gdabecf \\
$1:$ gdaebfc & $1:$ gcdaebf \\
$1:$ gcdeafb & $1:$ gbcdeaf \\
$1:$ gbcedfa & \\
\hline \hline
\end{tabular}

\begin{tabular}{c|c|c|c|c|c|c|c|}
\multicolumn{1}{c|}{ Pairwise comparisons } \\
\hline \hline vs & $a$ & $b$ & $c$ & $d$ & $e$ & $f$ & $g$ \\
\hline$a$ & - & 7 & 5 & 3 & 5 & 8 & 0 \\
\hline$b$ & 2 & - & 7 & 5 & 5 & 8 & 0 \\
\hline$c$ & 4 & 2 & - & 7 & 5 & 8 & 0 \\
\hline$d$ & 6 & 4 & 2 & - & 5 & 7 & 0 \\
\hline$e$ & 4 & 4 & 4 & 4 & - & 9 & 0 \\
\hline$f$ & 1 & 1 & 1 & 2 & 0 & - & 0 \\
\hline$g$ & 9 & 9 & 9 & 9 & 9 & 9 & - \\
\hline \hline
\end{tabular}

The reader can check that $\mathcal{Y} C_{2}(\pi)=\{\{a, g\},\{e, g\}\}$. When we replicate the electorate 2 times, we get $y C_{2}(2 \pi)=\{e, g\}$; committee $\{a, g\}$ is no more elected. Thus, the YC rule is not homogeneous.

\footnotetext{
${ }^{15}$ Thanks to an anonymous referee for pointing this out.
} 


\subsection{The reinforcement requirement}

Introduced by Smith (1973), the reinforcement axiom ${ }^{16}$ requires that when an electorate is divided in two groups of voters and that the voting outcome is the same for both groups, this outcome will remain unchanged when both groups of voters are merged.

Assume that the set of voters $N$ is divided in two disjoints groups $N_{1}$ and $N_{2}\left(N_{1} \cap N_{2}=\emptyset\right.$ and $\left.N_{1} \cup N_{2}=N\right)$. We denote by $\pi_{i}$ the preference profile associated with group $N_{i}(i=1,2)$ such that $\pi_{1} \cup \pi_{2}=\pi$. Given that $g$ is the size of the committee to be elected and $V$ a stable rule, we denote by $\mathcal{V}_{g}\left(\pi_{i}\right)$ and $\mathcal{V}_{g}(\pi)$ the set of the elected committees with the profiles $\pi_{i}$ and $\pi$ respectively.

Definition 4. (Reinforcement criterion) The stable rule $V$ satisfies the reinforcement axiom if and only if

$$
\mathcal{V}_{g}\left(\pi_{1}\right) \cap \mathcal{V}_{g}\left(\pi_{2}\right) \neq \emptyset \Rightarrow \mathcal{V}_{g}(\pi)=\mathcal{V}_{g}\left(\pi_{1}\right) \cap \mathcal{V}_{g}\left(\pi_{2}\right)
$$

Definition 5. (Weak reinforcement criterion) The voting rule $V$ satisfies the weak reinforcement axiom if and only if

$$
\forall C \in \mathcal{V}_{g}\left(\pi_{1}\right) \cap \mathcal{V}_{g}\left(\pi_{2}\right) \Rightarrow C \in \mathcal{V}_{g}(\pi)
$$

We know from the results of Young (1975) and Moulin (1988) that no Condorcet consistent rules satisfies reinforcement ${ }^{17}$. As a corollary, Theorem 6 tells us that every stable rule fails the reinforcement criterion and give more precisions on the stable rules analyzed in this paper.

Theorem 6. Every stable rule fails the reinforcement criterion. More, with at least five voters and at least three candidates, the KR rule, the DR rule, the MDC rule, the SEO rule, the NED rule and the YC rule fail the reinforcement criterion.

The proofs of Theorem 6 is provided in Appendix.

In Table 1, we report the results of our evaluation of the six stable rules analyzed here; in this table, a "yes" means that the voting rule meets the supposed property and a "no" if it does not.

Table 1: Stable rules and their properties

\begin{tabular}{c|c|c|c|c|c|c|c|c|}
\cline { 3 - 9 } \multicolumn{2}{c|}{} & \multicolumn{9}{c|}{ Criteria } & \multicolumn{2}{c|}{$\begin{array}{c}\text { Condorcet } \\
\text { winner }\end{array}$} & $\begin{array}{c}\text { Condorcet } \\
\text { loser }\end{array}$ & $\begin{array}{c}\text { Candidate } \\
\text { Monotonicity }\end{array}$ & $\begin{array}{c}\text { Membership } \\
\text { Monotonicity }\end{array}$ & Reinforcement & Homogeneity & Pareto \\
\hline \multirow{4}{*}{ Rules } & KR & no & no & yes & no & no & yes & yes \\
\cline { 2 - 10 } & DR & no & no & yes & no & no & no & yes \\
\cline { 2 - 10 } & SEO & no & no & yes & no & no & yes & yes \\
\cline { 2 - 10 } & YC & no & no & yes & no & no & no & yes \\
\cline { 2 - 9 } & MDC & yes & yes & yes & no & no & yes & yes \\
\cline { 2 - 9 } & NED & yes & yes & yes & no & no & yes & yes \\
\hline
\end{tabular}

$K R=$ Kemeny-Ratliff rule; $D R=$ Dodgson-Ratliff rule; $S E O=$ Minimal Size of External Opposition rule; $Y C=$ Young Condorcet rule; $M D C=$ Minimal Deletion of Candidates rule; $N E D=$ Minimal Number of External Defeats rule.

It comes that the KR rule, the DR rule, the SEO rule and the YC rule do not behave as the voting rules they are derived from. It comes from Table 1 that apart from the fact that the NED

\footnotetext{
${ }^{16}$ This criterion is called the Separability axiom in Smith (1973), Consistency axiom in Young (1975), Reinforcement axiom in Myerson (1995), etc.

${ }^{17}$ Courtin et al. $(2012,2014)$ suggested a slightly different version of this result; they found that with at least seven voters and at least 3 candidates, all the Condorcet consistent rules they focused on do not meet the reinforcement criterion.
} 
and the MDC rules violate the reinforcement criterion and the monotonicity criterion, they tend to stand out from the others rules: they satisfy more criteria than the others and they are the only rules that meet both the Condorcet Winner and the Condorcet Loser criteria.

\section{Concluding remarks}

In this paper, we have proposed two new voting rules for electing the Weak Condorcet Committee (WCC) when it exists. The first one, the YC (Young-Condorcet) rule is derived from the Young rule; it selects the fixed-size committee(s) that need(s) the fewest number of deletions of voters to become a WCC. The other new stable rule we proposed, the MDC (Minimal number of Deletion of Candidates) rule selects the fixed-size committee(s) that need(s) the fewest number of deletions of candidates to become a WCC. These new stable rules are added to the four other rules already suggested in the literature by Ratliff (2003) and Coelho (2004): the KR rule (derived from the Kemeny rule), the DR rule (adapted from the Dodgson rule), the SEO rule (adapted from the Maximin rule) and the NED rule.

We have pointed out that there is no formal connection between the stable rules we analyzed and the classical rules they are adapted from: one cannot find a generic way to transform a singlewinner rule to a committee rule such that when applying this transformation to the Kemeny rule, the Dodgson rule and so on, we get the corresponding stable rule. What we know is that it is only for voting situations with three candidates and for two-member committees that the outcome sets of the stable rules always have at least one committee in common. As nothing is known in the literature about the properties of all the stable rules proposed, we have tried to evaluate our stable rules on the basis of some appealing properties of voting rules: the Condorcet winner criterion, the Condorcet loser criterion, the Pareto criterion, the homogeneity criterion, the monotonicity criterion and the reinforcement requirement. We showed that they all fail the property of reinforcement and they are non-monotonic. Among our stable rules, only the NED and the MDC rules always elect the Condorcet winner when he exists and they always prevent the election of the Condorcet loser when he exists. The NED and the MDC rules tend to stand out from the others. Nonetheless, we admit that the criteria used here to evaluate the stable rules are not enough to draw an hierarchy of the stable rules. We need a larger range of criteria to make accurate conclusions. A possibility of making the results more general would be to show that certain properties hold for whole classes of committee voting rules as in Elkind et al. (2015b) since some of our rules have a flavor of distance-rationalization. A general results about the properties of such rules would be arguably more interesting than checking properties ad hoc rule by rule.

\section{Appendices : remaining proofs}

\section{Appendix 1: Proof of Theorem 3}

Consider a profile $\pi$ with $m \geq 3$ and $1 \leq g \leq m-1$ such that $C_{g}=\emptyset$. Assume that for $x, y \in A, x$ Pareto-dominates $y$ and that there is a committee $C \in \mathcal{N E D}_{g}(\pi)$ such that $y \in C$ and $x \notin C$. We define a committee $C^{\prime}=C \backslash\{y\} \cup\{x\}$. We also define two subsets $Z=C \cap C^{\prime}$ and $Q=A \backslash\left\{C \cup C^{\prime}\right\}$.

We denote by $\operatorname{def}(y / Q)$ the total number of defeats of candidate $y$ versus candidates in $Q$. Similarly, we define $\operatorname{def}(Z / Q)$ the total number of defeats of candidates in $Z$ versus those in $Q$. 
In the same manner we define $\operatorname{def}(x / y)$ and $\operatorname{def}(Z / y)$. We get

$$
\begin{aligned}
\operatorname{NED}(\pi, C) & =\operatorname{def}(y / x)+\operatorname{def}(y / Q)+\operatorname{def}(Z / x)+\operatorname{def}(Z / Q) \\
\operatorname{NED}\left(\pi, C^{\prime}\right) & =\operatorname{def}(x / Q)+\operatorname{def}(Z / Q)+\operatorname{def}(Z / y)
\end{aligned}
$$

By definition, $\operatorname{def}(y / x)=1$ and we get

$$
\operatorname{NED}(\pi, C)-\operatorname{NED}\left(\pi, C^{\prime}\right)=1+\operatorname{def}(y / Q)-\operatorname{def}(x / Q)+\operatorname{def}(Z / x)-\operatorname{def}(Z / y)
$$

Notice that as $x$ Pareto-dominates $y, y M(\pi) z \Rightarrow x M(\pi) z$ for all $z \in Z$ and $q M(\pi) x \Rightarrow q M(\pi) y$ for all $q \in Q$; so, $\operatorname{def}(Z / x)-\operatorname{def}(Z / y) \geq 0$ and $\operatorname{def}(y / Q)-\operatorname{def}(x / Q) \geq 0$. It follows that $\operatorname{NED}(\pi, C)>\operatorname{NED}\left(\pi, C^{\prime}\right)$ and this contradicts that $C$ is elected.

As $x$ Pareto-dominates $y$, it is obvious that $\operatorname{SEO}(\pi, C)=Y C(\pi, C)=n$. Assume there is a committee $C$ " consisting of top $g$ candidates of some voters for which $\operatorname{SEO}\left(\pi, C^{\prime \prime}\right) \leq n-1$ and $\mathrm{YC}\left(\pi, C^{\prime \prime}\right) \leq n-1$. So, $\operatorname{SEO}(\pi, C)>\operatorname{SEO}(\pi, C$ ") and $Y C(\pi, C)>Y C(\pi, C$ ") $:$ the committee $C$ is not elected with the SEO rule nor with the YC rule.

Let us assume that $C \in \mathcal{M D C}_{g}(\pi)$. We denote by $Q_{x}$ a subset of $Q$ such that $Q_{x}=\{q \in Q$ : $q M(\pi) x\}$. Similarly, we define $Q_{y}=\{q \in Q: q M(\pi) y\}$ and $Q_{z}=\{q \in Q: q M(\pi) z, z \in Z\}$. The MDC-scores are as follows:

$$
\begin{aligned}
\operatorname{MDC}(\pi, C) & =\left|Q_{y} \cup Q_{z}\right|+1 \\
\operatorname{MDC}\left(\pi, C^{\prime}\right) & = \begin{cases}\left|Q_{x} \cup Q_{z}\right|+1 & \text { if } y \text { defeats some } z \text { in } Z \\
\left|Q_{x} \cup Q_{z}\right| & \text { if } y \text { defeats no } z \text { in } Z\end{cases}
\end{aligned}
$$

As $x$ Pareto-dominates $y$, we get $Q_{x} \subseteq Q_{y}$ and $\left|Q_{x} \cup Q_{z}\right|-\left|Q_{y} \cup Q_{z}\right| \leq 0$. So, in all cases, we get $\operatorname{MDC}(\pi, C)-\operatorname{MDC}\left(\pi, C^{\prime}\right) \geq 0$. This contradicts that $C$ is elected while this is not also the case for $C^{\prime}$.

With the KR rule, the scores for committees $C$ and $C^{\prime}$ are:

$$
\begin{aligned}
\mathrm{KR}(\pi, C) & =n+\sum_{z \in Z} \max \left\{0, n_{x z}-n_{z x}\right\}+\sum_{q \in Q, z \in Z} \max \left\{0, n_{q z}-n_{z q}\right\}+\sum_{q \in Q} \max \left\{0, n_{q y}-n_{y q}\right\} \\
\mathrm{KR}\left(\pi, C^{\prime}\right) & =\sum_{q \in Q} \max \left\{0, n_{q x}-n_{x q}\right\}+\sum_{q \in Q, z \in Z} \max \left\{0, n_{q z}-n_{z q}\right\}+\sum_{z \in Z} \max \left\{0, n_{y z}-n_{z y}\right\}
\end{aligned}
$$

Then we get

$\operatorname{KR}(\pi, C)-\operatorname{KR}\left(\pi, C^{\prime}\right)=n+\sum_{z \in Z} \max \left\{0, n_{x z}-n_{z x}\right\}+\sum_{q \in Q} \max \left\{0, n_{q y}-n_{y q}\right\}-\sum_{q \in Q} \max \left\{0, n_{q x}-n_{x q}\right\}-\sum_{z \in Z} \max \left\{0, n_{y z}-n_{z y}\right\}$

As $x$ Pareto-dominates $y$, we end with $\sum_{q \in Q} \max \left\{0, n_{q y}-n_{y q}\right\} \geq \sum_{q \in Q} \max \left\{0, n_{q x}-n_{x q}\right\}$ and also $\sum_{z \in Z} \max \left\{0, n_{x z}-n_{z x}\right\} \geq \sum_{z \in Z} \max \left\{0, n_{y z}-n_{z y}\right\}$; it follows that $\mathrm{KR}(\pi, C)>\operatorname{KR}\left(\pi, C^{\prime}\right)$. So, the $\mathrm{KR}$ rule satisfies the Pareto condition.

Let us now prove that the DR rule meets the Pareto criterion. Assume that committee $C$ is elected while this is not the case for committee $C^{\prime}$. Given $x M(\pi) y$ we denote by $N_{y x}$ the smallest number of adjacent swaps we need in the individual rankings to get $y M(\pi) x$ or $x T(\pi) y$. In the 
same way we define $N_{z x}$ and $N_{z y}$ for $z \in Z ; N_{x Q}, N_{y Q}$ and $N_{z Q}$ for $q \in Q$. When computing the DR-scores for committees $C$ and $C^{\prime}$ we get

$$
\begin{aligned}
\operatorname{DR}(\pi, C) & =N_{y x}+\sum_{z \in Z} N_{z x}+\sum_{q \in Q} N_{y q}+\sum_{q \in Q, z \in Z} N_{z q} \\
\operatorname{DR}\left(\pi, C^{\prime}\right) & =\sum_{z \in Z} N_{z y}+\sum_{q \in Q} N_{x q}+\sum_{q \in Q, z \in Z} N_{z q}
\end{aligned}
$$

It follows that $\mathrm{DR}(\pi, C)-\mathrm{DR}\left(\pi, C^{\prime}\right)=N_{y x}+\sum_{z \in Z} N_{z x}+\sum_{q \in Q} N_{y q}-\sum_{z \in Z} N_{z y}-\sum_{q \in Q} N_{x q}$.

As $x$ Pareto-dominates $y$, it comes that $\sum_{z \in Z} N_{z x} \geq \sum_{z \in Z} N_{z y}$ and that $\sum_{q \in Q} N_{y q} \geq \sum_{q \in Q} N_{x q}$. So, we get $\mathrm{DR}(\pi, C)-\mathrm{DR}\left(\pi, C^{\prime}\right)>0 \Rightarrow \operatorname{DR}(\pi, C)>\operatorname{DR}\left(\pi, C^{\prime}\right)$. This contradicts that committee $C$ is elected: the DR rule satisfies the Pareto criterion.

\section{Appendix 2: Proof of Theorem 4}

Consider a profile $\pi$ with at least three candidates. For simplicity, let us denote by $V$ any of our stable rules, by $V(\pi, C)$ the score of a committee $C$ under $V$ and by $\mathcal{V}_{g}(\pi)$ the set of elected committees.

- Our stable rules meet the Candidate monotonicity criterion.

Let us denote by $C^{x}$ the set of committees containing $x$. Assume that some voters lift up candidate $x$ in their rankings without changing anything else. It is obvious that for $y \neq x$, we get $n_{y x}\left(\pi^{\prime} \leq\right.$ $n_{y x}(\pi)$ and $n_{x y}\left(\pi^{\prime}\right) \geq n_{x y}(\pi)$ in the new profile $\pi^{\prime}$. Also, we get (i) $V\left(\pi^{\prime}, C\right) \leq V(\pi, C)$ for all $C \in C^{x}$ and (ii) $V\left(\pi^{\prime}, C\right) \geq V(\pi, C)$ for all $C \notin C^{x}$. Let us take a given committee $C \in C^{x}$ such that $C \in \mathcal{V}_{g}(\pi)$. Assume that $V$ does not meet the Candidate monotonicity criterion. This means that no committee in $C^{x}$ is elected after the lifting-up: there is a committee $C^{\prime} \notin C^{x}$ such that $V\left(\pi^{\prime}, C^{\prime}\right)<V\left(\pi^{\prime}, C\right)$. By (i) and (ii), we end with

$$
V\left(\pi, C^{\prime}\right) \leq V\left(\pi^{\prime}, C^{\prime}\right)<V\left(\pi^{\prime}, C\right) \leq V(\pi, C) \Rightarrow V\left(\pi, C^{\prime}\right)<V(\pi, C): \text { this contradicts } C \in \mathcal{V}_{g}(\pi)
$$

For all $C \in C^{x}$, there is an incompatibility between $C \in \mathcal{V}_{g}(\pi)$ and the violation of the Candidate monotonicity criterion. Thus, our stable rules meet the Candidate monotonicity criterion.

- Our stable rules do not meet the Membership Monotonicity criterion.

Notice that in case of the existence of a WCC, a lifting up of one of its member cannot be harmful. So, for the proof we only need to consider the cases where there is no WCC. For the sake of simplicity, we first focus on the cases where $m=3$ and $g=2$. We consider the following profile with three candidates $\{a, b, c\}$ and $3 k+2 p$ voters; $k$ is an integer greater or equal to 1 and $p$ is a nonnegative integer ${ }^{18}$.

\begin{tabular}{llll}
\hline \hline$k+p: a b c$ & $k: c a b$ & $p: c b a$ & $k: b c a$ \\
\hline
\end{tabular}

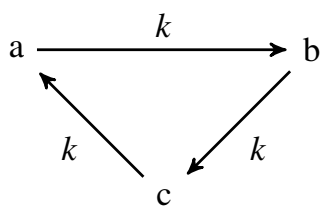

\footnotetext{
${ }^{18}$ With $n=3 k+2 p$, we cover the cases with odd and even sizes of the electorate.
} 
The reader can check that each of our stable rules will select $\{\{a, b\},\{a, c\},\{b, c\}\}$ for $g=2$. Assume that the $k$ voters with $b c a$ change to $c b a$; this leads to what follows

\begin{tabular}{llll}
$k+p: a b c$ & $k: c a b$ & $p: c b a$ & $k: c b a$ \\
\hline
\end{tabular}

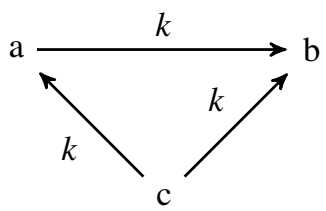

The committee $\{a, c\}$ becomes a WCC and is chosen by each of our stable rules. Candidate $c$ belongs to committee $\{b, c\}$ and he has benefited from a lifting up that has pushed committee $\{b, c\}$ out of the original set of winners. So, none of our stable rules meets the Membership Monotonicity criterion. To extend the proof to voting situations with more than three candidates, we just need to reconsider our original profile with $\{a, b, c\}$ and to add new candidates all ranked under candidates $a, b$ and $c$.

\section{Appendix 3: Proof of Theorem 6}

Let us consider a situation with three candidates ${ }^{19} A=\{a, b, c\}$. Let us assume that the size of the electorate is $n=3 p+2 k$ with $p$ and $k$ two positive integers $(p \geq 1, k \geq 1)$. This electorate is divided into two groups as follows.

\begin{tabular}{c}
$\pi_{1}$ \\
\hline \hline$k: a b c \quad k: a c b$ \\
\hline \hline
\end{tabular}

\begin{tabular}{lll}
\multicolumn{3}{c}{$\pi_{2}$} \\
\hline \hline$p: a c b$ & $p: b a c$ & $p: c b a$ \\
\hline \hline
\end{tabular}

The reader can check that, each of our stable rules $V$ leads to the same outcome. For each of the groups of the electorate, we get the corresponding outcomes sets:

$$
\mathcal{V}_{2}\left(\pi_{1}\right)=\{\{a, b\},\{a, c\}\}, \mathcal{V}_{2}\left(\pi_{2}\right)=\{\{a, b\},\{a, c\},\{b, c\}\} \text { and } \mathcal{V}(\pi)=\{a, c\}
$$

We notice that it is always possible to find $k$ and $p$ such that $\mathcal{V}_{2}\left(\pi_{1}\right) \cap \mathcal{V}_{2}\left(\pi_{2}\right) \neq \emptyset$ but when the two groups merge, committee $\{a, b\}$ is no longer appointed. So, when the number of voters is at least equal to five, all our stable rules fail the reinforcement condition.

We have to point that with $n=3 p+2 k(p \geq 1, k \geq 1)$, the case where $n=6$ is not covered. For $n=6$, we have used a complete enumeration procedure and we found that with three candidates it is only possible for the NED rule and the MDC rule to construct two profiles $\left(\pi_{1}\right.$ and $\left.\pi_{2}\right)$ such that when we merge the groups they fail the reinforcement criterion while this is not possible for the KR rule, the DR rule, the SEO rule and the YC rule. But for $n=6$ and four candidates, it is possible to construct profiles $\left(\pi_{1}\right.$ and $\left.\pi_{2}\right)$ such that when we merge the groups, the KR rule, the $\mathrm{DR}$ rule, the SEO rule and the YC rule fail the reinforcement criterion.

Acknowledgements. The author is grateful to the Editor and to two anonymous reviewers for their valuable and useful comments. The author would thank Vincent Merlin, Bernard Grofman and Ashley Piggins. Particular thanks to Hans Peters for all the discussions while I was visiting

\footnotetext{
${ }^{19}$ We can get the proof for voting profiles with more than three candidates; we just need to construct new profiles from those used here by adding as many candidates as we wish, all ranked after $a, b$ and $c$
} 
the Maastricht university under the STSM COST Action IC1205. Thanks also to Reiko Gotoh and the members of the Institute of Economic Research-Hitotsubashi University (Japan) for their remarks and suggestions. Thanks to all the participants to the session "Topics in Voting" at the 2015 European Public Choice Society meetings in Groningen (The Netherlands).

Barberà S. and Coelho D. (2008) How to choose a non-controversial list with $k$ names. Social Choice and Welfare 31: 79-96.

Bartholdi J., Tovey C.A. and Trick M.A. (1989) Voting schemes for which it can be difficult to tell who won the election. Social Choice and Welfare 6(3): 157-165.

Betzler N., Guo J. and Niedermeier R. (2010) Parameterized computational complexity of Dodgson and Young elections. Information and Computation 208(2): 165-177.

Black D. (1958) The Theory of Committees and Elections. Cambridge University Press, Cambridge.

Brams S.J (2008) Mathematics and Democracy : Designing Better Voting and Fair- Division Procedures. Princeton University Press.

Brams S.J, Kilgour D.M and Sanver M.R. (2005) A minimax procedure for electing committees. Public Choice, 132(34): 401-420.

Caragiannis I., Covey J., Feldman M., Christopher M. Homan C., Kaklamanis C., Karanikolas N., Procaccia A.D and Rosenscheiny J.S (2012) On the Approximability of Dodgson and Young Elections. Artificial Intelligence 187-188: 31-51.

Chamberlin J.R. and Courant P.N. (1983) Representative Deliberations and Representative Decisions : Proportional Representation and the Borda Rule. American Political Science Review, 77(3): 718-733.

Coelho D. (2004) Understanding, evaluating and selecting voting rules through games and axioms. Phd Thesis, Universitat Autonoma de Barcelona. Available at http://ddd.uab.es/record/36576? ln=en.

Marquis de Condorcet (1785) Essai sur l'Application de l'Analyse à la Probabilité des Décisions Rendues à la Pluralité des Voix. Paris.

Courtin S., Mbih B. and Moyouwou I. (2012) Are Condorcet procedures so bad according to the reinforcement axiom? Thema Working paper no 2012-37, Cergy Pontoise.

Courtin S., Mbih B. and Moyouwou I. (2014) Are Condorcet procedures so bad according to the reinforcement axiom? Social Choice and Welfare 42: 927-940.

Darmann A. (2013) How hard is it to tell which is a Condorcet committee? Mathematical Social Sciences 66(3): 282-292.

Dodgson C.L (1885a) The principles of parliamentary representation: Postscript to Supplement, E. Baxter Publisher, Oxford.

Dodgson C.L (1885b) The principles of parliamentary representation: Supplement, E. Baxter Publisher, Oxford.

Dodgson C.L (1884) The principles of parliamentary representation. Harrison and Sons Publisher, London.

Dodgson C.L. (1876) A Method of Taking Votes on More than Two Issues. Clarendon Press, Oxford.

Dummett M. (1984) Voting Procedures. Oxford, Clarendon Press.

Elkind E., Lang J. and Saffidine A. (2011) Choosing collectively optimal sets of alternatives based on the Condorcet criterion. In IJCAI11 pp. 186-191.

Elkind, E., Faliszewski P., Skowron, P. and Slinko A. (2014) Properties of multiwinner voting rules. In Proc. of the 13th AAMAS Conference, 53-60.

Elkind E., Lang J. and Saffidine A. (2015a) Condorcet winning sets. Social Choice and Welfare 44(3): 493-517.

Elkind, E., Faliszewski P. and Slinko A. (2015b) Distance rationalization of voting rules. Social Choice and Welfare 45(2): $345-377$

Felsenthal D.S. (2012) Review of Paradoxes Afflicting Procedures for Electing a Single Candidate in Electoral Systems : Paradoxes, Assumptions, and Procedures, Studies in Choice and Welfare, Felsenthal, DS and Machover, M. (Eds.), Springer Berlin Heidelberg.

Fishburn P.C (1981) An analysis of simple voting systems for electing committees. SIAM Journal on Applied Mathematics 41: 499-502

Fishburn P.C. (1977) Condorcet social choice functions. SIAM Journal on Applied Mathematics 33: 469-489.

Gerhlein W.V. and Fishburn P.C. (1976) The probability of the paradox of voting: A computable solution. Journal of Economic Theory 13: 14-25.

Gerhlein W.V. (1985) The Condorcet criterion and committee selection. Mathematical Social Sciences 10: 199-209.

Good I.J. (1971) A note on Condorcet sets. Public Choice 10(1): 97-101.

Good I.J and Tideman T. N (1976) A principle for selecting a representative committee. Mimeo, Virginia Polytechnic Institute and State University.

Hemaspaandra E., Hemaspaandra L. and Rothe J. (1997) Exact analysis of Dodgson elections: Lewis Carroll's 1876 voting system is complete for parallel access to NP. Journal of the ACM 44(6): 806-825. 
Hill I.D (1988) Some aspects of elections: To fill one seat or many. Journal of the Royal Statistical Society 151: 243-275

Kamwa E. (2016) Stable Rules for Electing Committees and Divergence on Outcomes. Group Decision and Negotiation, doi:10.1007/s10726-016-9504-8.

Kamwa E. (2014) Essais sur les modes de scrutins et la sélection des comités. PhD thesis, Université de Caen.

Kamwa E. (2013a) The Kemeny rule and committee elections. Economics Bulletin 33(1): 648-654

Kamwa E. (2013b) The increasing committee size paradox with a small number of candidates. Economics Bulletin 33(2): 967-972.

Kamwa E. and Merlin V. (2013c) Coincidence of Condorcet committees. Mimeo CREM Working Paper, University of Caen.

Kaymak B. and Sanver M.R. (2003) Sets of alternatives as Condorcet winners. Social Choice and Welfare 20: $477-494$.

Kemeny J. (1959) Mathematics without numbers. Daedalus 88: 571-591.

Kemeny J. and Snell I. (1960) Mathematical Models in the Social Sciences. Boston: Ginn.

Kilgour D.M., Brams S.J and Sanver M.R (2006) How to elect a representative committee using approval balloting. In B. Simeone and F. Pukelsheim, editors, Mathematics and Democracy : Recent Advances in Voting Systems and Collective Choice. Springer Heidelberg.

Kramer G.H. (1977) A dynamical model of political equilibrium. Journal of Economic Theory 16: 310-334.

Miller N.R. (1980) A New Solution Set for Tournaments and Majority Voting: Further Graph-Theoretical Approaches to the Theory of Voting. American Journal of Political Science 24(1): 68-96.

Mitchell D.W. and Trumbull W.N. (1992) Frequency of paradox in a common Nwinner voting scheme. Public Choice 73(1): 55-69.

Monroe B.L (1995) Fully proportional representation. American Political Science Review 89(4): 925-940.

Moulin H. (1988) Axioms of cooperative decision making. Cambridge Press, Cambridge.

Myerson R.B. (1995) Axiomatic derivation of scoring rules without the ordering assumption. Social Choice and Welfare 12: $59-74$.

Nurmi H.(2004) A comparison of some distance-based choice rules in ranking environments. Theory and Decision 57: $5-24$.

Nurmi H.(1987) Comparing Voting Systems D. Reidel Publishing Company.

Ratliff T.C. (2003) Some startling inconsistencies when electing committees. Social Choice and Welfare 21: $433-454$

Richelson J.T. (1979) A comparative-analysis of social choice functions, I, II, III : A summary. Systems Research 24: 355.

Richelson J.T. (1981) A comparative analysis of social choice functions IV. Systems Research 26: 346-353.

Rothe J., Spakowski H. and Vogel J. (2003) Exact complexity of the winner problem for young elections. Theory of Computing Systems, 36(4): 375-386.

Simpson P. (1969) On defining areas of voter choice. Quarterly Journal of Economics 83: 478-490.

Smith J.H. (1973) Aggregation of preferences with variable electorate. Econometrica 41: 1027-1041.

Staring M. (1986) Two paradoxes of committee elections. Mathematics Magazine 59(3): 158-159.

Sterne S. (1871) On representative government and personal reprsentation. Philadelphia: J.B. Lippincott.

Tideman T.N. (1987) Independence of clones as a criterion for voting rules. Social Choice and Welfare 4: 185-206.

Tullock G. (1967) Toward a mathematics of politics. Ann Arbor : University of Michigan Press.

Young H.P. (1975) Social Choice scoring functions. SIAM Journal of Applied Mathematics 28: 824-838.

Young H.P. (1977) Extending Condorcet's rule. Journal of Economic Theory 16: 335-353.

Young H.P and Levenglick A. (1978) A consistent extension of Condorcet's election principle. SIAM Journal of Applied Mathematics 35: 285-300. 